\section{How to treat proximal and middle one-third humeral shaft fractures: The role of helical plates}

\author{
Giulia Nicolaci, ${ }^{1}$ Nicola Lollino ${ }^{2}$ \\ ${ }^{1}$ Department of Orthopaedics and \\ Traumatology, University of Turin, \\ Turin; ${ }^{2}$ Orthopaedics and Traumatology \\ Unit, E. Agnelli Hospital, Pinerolo, Italy
}

\begin{abstract}
Complex proximal third diaphyseal humeral fractures are uncommon patterns of injury mainly caused by high energy trauma. The anatomical shape of the humerus, the presence of the deltoid tuberosity and the close proximity of the radial nerve into the radial groove represent challenge elements to deal with. Historically, straight plates were manually twisted; subsequently, helical plates created for other anatomical sites (as distal tibia) were used in humeral fractures. In both these experiences surgeons observed several disadvantages. More recently, dedicated helical plates have been created. In this study, we expose our surgical technique for using helical humeral plates (A.L.P.S. ${ }^{\circledR}$ Proximal Humeral Plating System, Zimmer Biomet), with its advantages and operative recommendation. From 2019 to 2021, nine patients who were admitted to our institution for humeral fractures involving the proximal third diaphysis have been treated with humeral helical plates. At one and six months after surgery, standard antero-posterior and lateral radiographs were obtained, and at last followup (fourteen months on average) clinical evaluation was performed through range of motion assessment, Constant score and DASH score questionnaires. At six months, all fractures have healed. At last follow-up (fourteen months on average, 6-22) the average range of motion were flexion $135^{\circ}$ $\left(90^{\circ}-180^{\circ}\right)$; abduction $124^{\circ}\left(85^{\circ}-180^{\circ}\right)$; external rotation $52^{\circ}\left(20^{\circ}-80^{\circ}\right)$, internal rotation at L3 (between scapulaetrochanter). Average Constant Shoulder Score was 70 (33-96), average Dash score was 21 (range 1,7-63). Three patients experienced temporary radial nerve palsy from injury, with subsequently improvement at EMG analysis within eight months from surgery. In our opinion this strategy avoids the deltoid tuberosity and reduces the risk of radial nerve injury, increasing the possibility of a rapid functional recovery after surgery.
\end{abstract}

\section{Introduction}

Humeral shaft fractures account for approximately $3 \%$ of all orthopaedic injuries. ${ }^{1}$ Most of the humeral shaft fractures can be treated nonoperatively: studies in literature reported excellent results with high rates of bone union and functionally and aesthetically acceptable residual deformities. ${ }^{1-3}$ Operative treatment has gained popularity for displaced, open or pathological fractures, fractures of the proximal or distal third of the shaft and fractures with ipsilateral brachial plexus or vascular injuries, and several options exist based on bone quality, type of fracture, its location, associated soft tissue injuries. ${ }^{1,4}$

Proximal and middle one-third diaphyseal humeral fractures, instead, are barely described in literature and controversies exist about the ideal treatment. Studies analyzing the intramedullary nail technique showed a higher risk of fixation failure in comminuted and osteoporotic bones and in fractures extending into the tuberosity or metaphysis; ${ }^{5,6}$ moreover, some clinical series reported debilitating shoulder complications due to nail insertion through the rotator cuff. ${ }^{1,4}$ Alternatively, locking plate fixation in humeral fractures has spread given its less amount of interference with elbow and shoulder function, rapid functional recovery and its stable fixation. ${ }^{5}$ However, in proximal and middle third diaphyseal fractures plates have some limitations: ${ }^{5}$ the radial nerve is at risk during the surgical approach at the middle diaphysis and detachment of the deltoid from its tuberosity is a common maneuver to accommodate the plate adequately, but it may slow functional recovery. ${ }^{7,8}$ The iatrogenic radial nerve injury is described in about $7 \%$ of cases $(2.7 \%-20 \%),{ }^{9}$ secondary to intraoperative traction rather than direct plating compression; this susceptibility is due to its fixed position in the sulcus radialis and its direct contact with the periosteum of the humerus after passing the medial intermuscular septum.

Regarding the deltoid detachment, in the past some authors have proposed manually twisting straight plates to avoid the deltoid insertion: despite satisfied results, it is unknown to what extent the twisting weaken the plate, and the twisted head may accommodate fewer screws for the humeral head. ${ }^{7-11}$ Other reports describe the application of helical plates on the humerus originally designed for other anatomical areas. ${ }^{5}$ The literature that deals with helical plates is however scarce. With our study we describe our experience using helical plates specifically designed for the humerus (A.L.P.S. ${ }^{\circledR}$ Proximal Humeral Plating
Giulia Nicolaci, Department of Orthopaedics and Traumatology, Università degli Studi di Torino, Via Gianfranco Zuretti 29, 10126 Torino, Italy

E-mail: giulia.nicolaci@edu.unito.it

Key words: Proximal third diaphyseal humeral fractures; complex humeral fractures; helical plates- deltoid tuberosity; surgical approach

Acknowledgments: We would like to express our thank to Zimmer Biomet s.r.l, Italy for the technological support during the operative phase of the procedures. Zimmer Biomet s.r.l, Italy gave the permission to publish the images.

Contributions: GN: conceptualization; investigation; writing; NL: conceptualization; project administration; supervision.

Conflict of interest: The authors declare no conflict of interest.

Ethics approval: All procedures performed in studies involving human participants were in accordance with the ethical standards of the institutional and national research committee and with the 1964 Helsinki declaration and its later amendments or comparable ethical standards.

Informed consent, consent to participate, consent to publish: Informed consent was obtained from all individual participants included in the study, who agreed to participate to the study and approved having their data published

Received for publication: 14 June 2021 Accepted for publication: 16 June 2021

This work is licensed under a Creative Commons Attribution NonCommercial 4.0 License (CC BY-NC 4.0).

(C) Copyright: the Author(s), 2021

Licensee PAGEPress, Italy

Surgical Techniques Development 2021; 10:9175 doi:10.4081/std.2021.9175

System, Zimmer Biomet) for managing proximal and middle one-third diaphyseal fractures, reporting clinical evaluation of patients based on the range of motion measurement, DASH Questionnaire and Constant score.

\section{Material and Methods}

From 2019 to 2021, we have evaluated a consecutive series of patients who were admitted to our institution for humeral fractures involving the proximal third diaphysis and caused by high energy traumas or falls. 
Exclusion criteria were open or pathological fractures, patients with multiple injuries, fractures involving only the proximal or distal humerus, non-displaced fractures, patients unfit for surgery or non-compliant with operative and post-operative protocol. Nine patients met inclusion criteria at first, but one was excluded due to SARSCoV2 infection after revision plate fixation for a failed nail treatment. The mean age was 70 (48-84); AP and lateral radiographs were obtained: most fractures were classified as $\mathrm{AO} 12 \mathrm{~A} 1 / 12 \mathrm{C} 2$ (three for each group); one case had a AO12B2 fracture type, and one fracture was evaluated as a AO12C3 type (Figure 1). Five patients showed proximal third diaphyseal fractures with extension to the metaphysis and the humeral head; the other three patients had fractures involving the medial third and distal shaft fractures.

In three subjects a complete radial nerve palsy was also present from injury (Figure 2). All patients received informed consent and agreed to participate in the study. After surgery, patients started gentle passive range of motion exercises of the shoulder and elbow the day after the operation, and they were followed up in the clinic for six months at least: at one and six months standard antero-posterior and lateral radiographs were obtained (Figure 3 and 4: X ray images at one and six months of followup). Fractures were reported as united if the patient reported no or mild pain with radiographically bridging callus in 3 of 4 cortices in AP/lateral views, no widening fracture gaps, no loss of fracture reduction, nor implant failure. At a mean time of fourteen months (6-22 months of follow-up), we performed a clinical evaluation with a range of movement measurements, and participants were asked to answer Constant score and DASH questionnaire (Table 1).

\section{Technique}

All cases were treated by the same surgeon with a deltopectoral approach extended distally along the anterolateral aspect of the diaphysis. After the incision of the deltopectoral fascia the surgeon identified and protected the cephalic vein, dissected soft tissue medializing the biceps muscle and deepened to the bone, using Hohmann retractors and periosteal elevators for exposition. Careful attention was made to preserve the deltoid insertion; we preferred placing retractors at this level and not proximally to avoid circumflex nerve damage. In some fracture extended proximally a

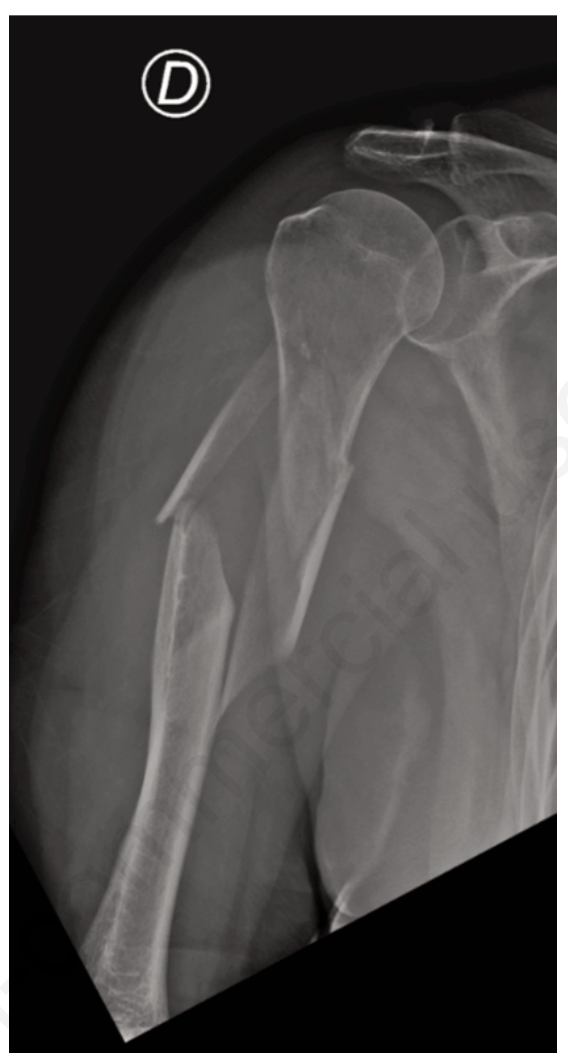

Figure 1. Example of fracture type included in the study. slight release of the pectoral major was necessary to attached reduction clamps firmly to bone. In cases with a rim fracture extended distally, the brachialis detachment might become necessary for correct reduction and plate positioning. The deltoid lever arm may be a contributing factor in fragment displacement, but the aim is to avoid deltoid

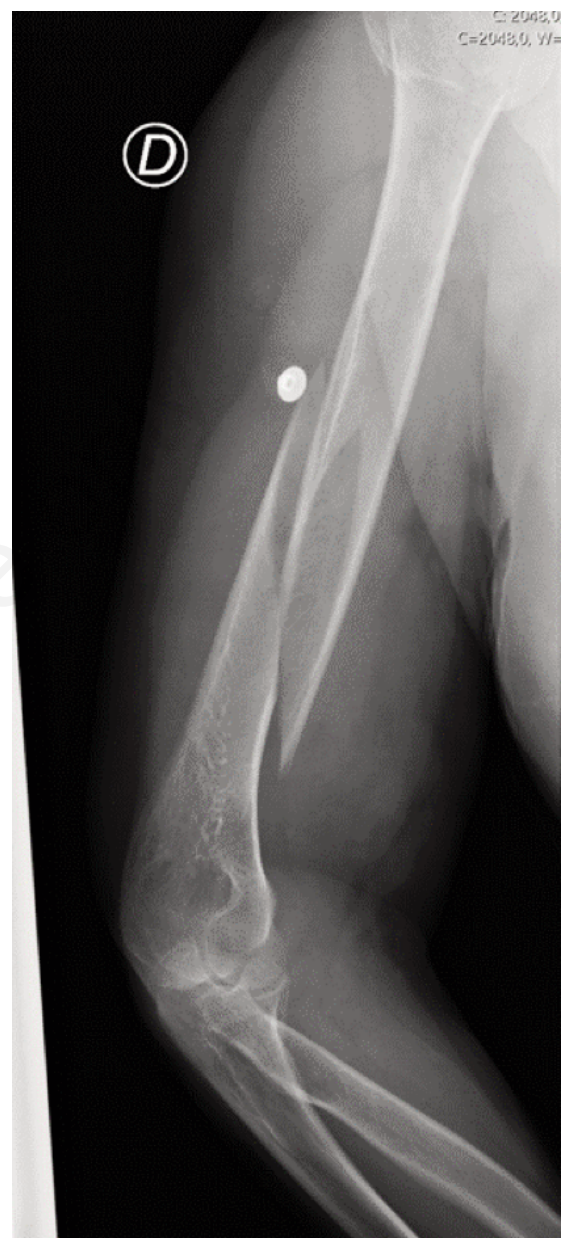

Figure 2. Humeral shaft and surgical neck fracture with radial palsy.

Table 1. Clinical evaluation of the aptients with a range of movement measurements, and participants' answers to Constant score and DASH questionnaires.

\begin{tabular}{|c|c|c|c|c|c|c|c|}
\hline Case & Sex/age & Mode of injury & A0 classification & Follow up months & ROM (Flex/abd/ER/R) & Constant score & Dash score \\
\hline 1 & $\mathrm{~F} / 72$ & Fall & $\mathrm{A} 012 \mathrm{C} 2$ & 16 & 100/90/45/LS junction & 80 & 15.8 \\
\hline 2 & $\mathrm{~F} / 48$ & Pedestrian & $\mathrm{AO} 12 \mathrm{C} 2$ & 12 & 180/160/60/L3 & 94 & 9,5 \\
\hline 3 & $\mathrm{~F} / 68$ & Fall & AO 12Al & 12 & 180/120/60/LS junction & 82 & 15.8 \\
\hline 4 & $\mathrm{M} / 76$ & Fall & A012B2 & & & & \\
\hline 5 & $\mathrm{~F} / 84$ & Fall & $\mathrm{AO} 12 \mathrm{C} 3$ & 6 & 160/170/60/L3 & 96 & 9,5 \\
\hline 6 & $\mathrm{~F} / 80$ & Fall & AO 12Al & 6 & 90/90/20/LS junction & 73 & 20 \\
\hline 7 & $\mathrm{~F} / 60$ & Fall & $\mathrm{AO} 12 \mathrm{~B} 2$ & 7 & 110/110/50/buttock & 58 & 42,5 \\
\hline 8 & $\mathrm{~F} / 75$ & Fall & $\mathrm{AO} 12 \mathrm{C} 2$ & 6 & 120/120/30/LS & 76 & 23,3 \\
\hline 9 & F/ 65 & Fall & $\mathrm{AO} 12 \mathrm{Al}$ & 4 & 180/160/45/L3 & 84 & 18,5 \\
\hline
\end{tabular}




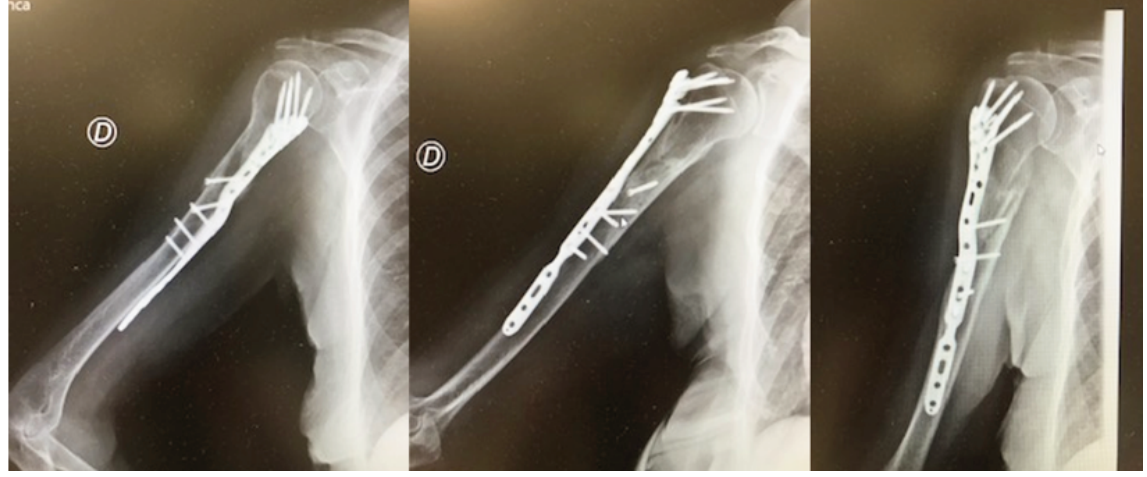

Figure 3. $\mathrm{X}$ ray images at one month of follow-up.

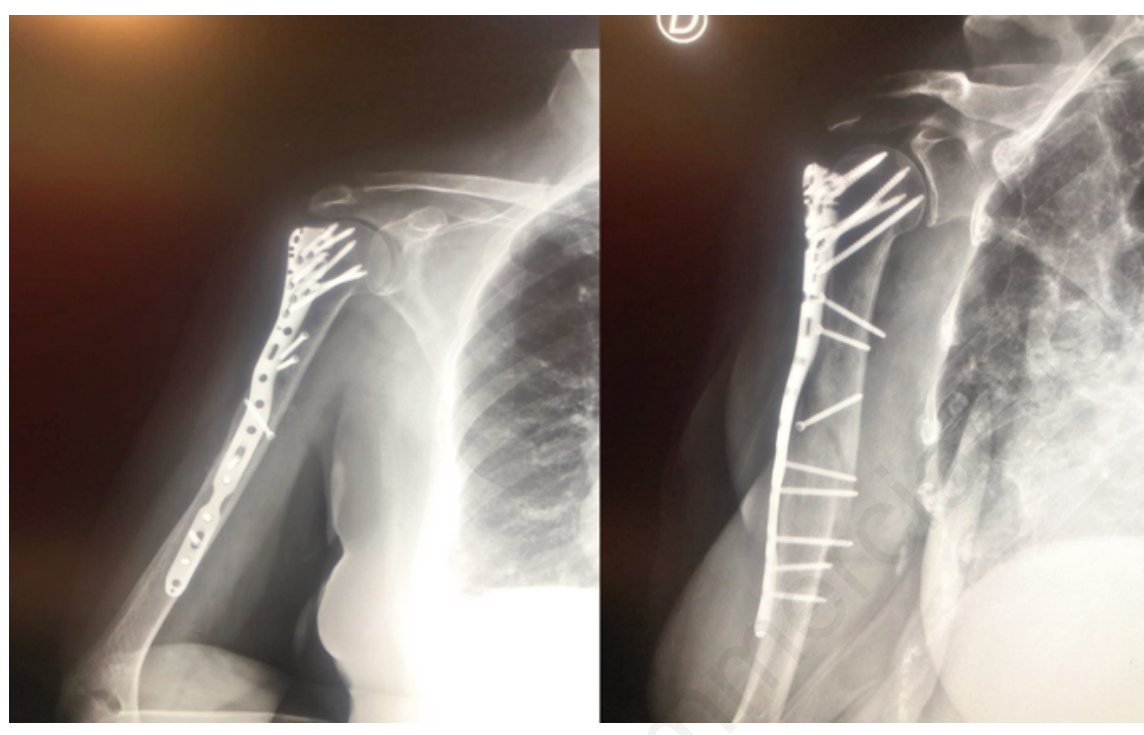

Figure 4. X ray images at six months of follow-up.

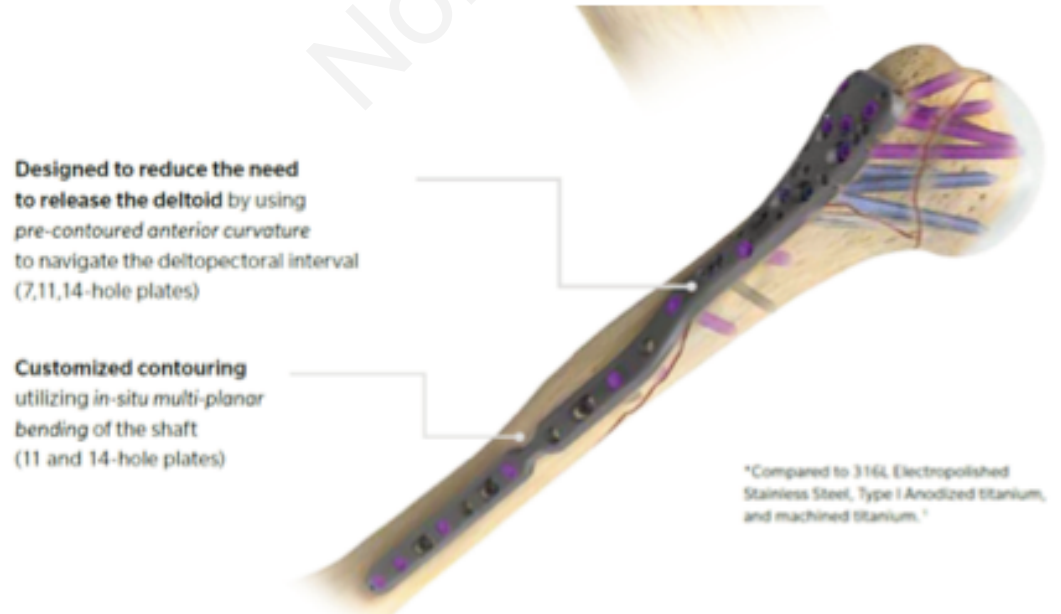

detachment. A partial arm abduction can help the surgeon obtain a correct reduction

Figure 5. A.L.P.S. ® Proximal Humeral Plating System, Zimmer Biomet - image by Zimmer Biomet. without violating the muscle. After temporary reduction with clamps and satisfactory fluoroscopic view, definite fixation was performed: in complex fractures cortical lag screws were employed to stabilize fragments; finally, a helical plate with angular stability (A.L.P.S. ${ }^{\circledR}$ Proximal Humeral Plating System, Zimmer Biomet) was positioned (Figure 5). This recently designed implant helps surgeons avoid complete deltoid detachment during the soft tissue dissection: the plate has a twist at $90-120 \mathrm{~mm}$ on average from greater tuberosity, resulting in a lateral position proximally, posterior to the biceps tendon, and an anterior position distally. Because of individual anatomical differences and to avoid manual correction of the twist, although possible, the surgeon paid attention to obtain the correct angulation at the deltoid insertion level at first and fix the proximal and distal segments with screws subsequently. We used angular stability fixation to minimize periosteal devascularization: the Zimmer system offers different screw options depending on bone quality: cortical and cancellous locking screws, low profile nonlocking screws, multi-directional locking screws. Non-locking and multi-directional screws may be an effective aid to obtain a better plate-bone congruence and fixation even in cases of suboptimal plating placement. The distal anterior placement reduces the risk of direct radial nerve conflict. The proximal humeral plate is available in two height options based on direct screw fixation of the greater tuberosity or not. The maximal length is 14 holes (227-234 mm). In almost all patients ( 23 out of 24 ) we used 11 or 14 hole-plates (Figure 6): the length

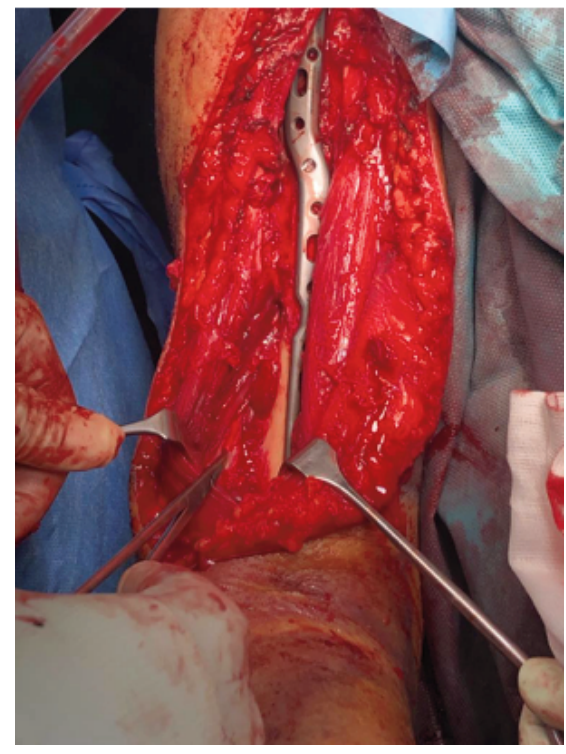

Figure 6. Plate fixation preserving deltoid insertion. 
and number of screws depended on fracture morphology, but we ensured two bicortical locking screws on each side at least, following the $\mathrm{AO}$ technical indications for these fractures. ${ }^{12}$ In none of our cases a bone graft was needed, but it could be a feasible option to add mechanical support in fractures with a significant bone loss.

We explored the radial nerve in cases with posttraumatic nerve palsy, and it was found continuous at the fracture site.

No differences in blood loss and operative time were recorded in the comparison between the application of helical and conventional implants.

\section{Results}

We finally evaluated eight patients; the average follow-up period was fourteen months (range 6-22). There were no cases of intraoperative or postoperative complications. In the three patients with preoperative radial nerve palsy, a near-to-complete recovery was reached in the first case after eight months and in the second case after six months. In the last patient, instead, a nervous deficit was still present at the last follow-up (eight months from injury), although with progressive improvement at EMG analysis.

All fractures healed uneventfully, as it was observed on radiographs at one and six months of follow-up (Figure 5-6). The average range of motion at the last follow-up were flexion $135^{\circ}\left(90^{\circ}-180^{\circ}\right)$; abduction $124^{\circ}\left(85^{\circ}-180^{\circ}\right)$; external rotation $52^{\circ}\left(20^{\circ}\right.$ $80^{\circ}$ ), internal rotation at L3 (between scapulae-trochanter). Average Constant Shoulder Score was 70 (33-96), average Dash score was 21 (range 1,7-63).

\section{Discussion}

Focusing on plating fixation in proximal humeral fractures, an advantage is the congruence between the bony shape and the plate: the flat surface of the great tuberosity allows for screw fixation of the neck and head of the humerus. ${ }^{5}$ Moving more distally, however, the presence of the deltoid insertion limits the placement of the plate on the lateral aspect of the bone. Due to this discordance, in conventional plating a partial or nearly complete detachment of the deltoid insertion is necessary. Moreover, a straight plate also induces undue stress shielding of the fractured bone, being positioned on the tensile side of the bone. ${ }^{5}$

Benninger and Meier ${ }^{13}$ performed minimally invasive plate placement on eight cadavers and reported some important observa- tions: the deltoid muscle has seven intramuscular tendons that create a V-shaped insertion, where the middle part is the weakest; a lateral plate position may pose radial nerve at risk; the deltopectoral approach is safer than a deltoid split for the axillary nerve. Robinson et al. ${ }^{14}$ and Dauwe et $a l .{ }^{15}$ in 2020 studied the risk of nerve damage: Robinson et al. ${ }^{14}$ described a higher risk of axillary nerve injury during the insertion of straight plates with the MIPO technique, while in a cadaveric study Dauwe et al. ${ }^{15}$ showed a lower mean platebone distance with helical plates than with straight models, an indirect measure of lower risk of axillary nerve elongation.

Since 1996 Yang examined this topic and in 2005 used pre-contoured helical plates to treat comminuted proximal humeral fractures: $^{8}$ although satisfactory recovery of shoulder range of motion, the author hypothesized that the action of twisting may weaken the plate and reduces the number of holes able to accommodate screws for proximal fixation. ${ }^{16}$ In 1999, Gill and Torchia described a case of humeral nonunion and brachial plexus managed with a spiral compression plate to preserve the deltoid muscle insertion, with the aim to promote early rehabilitation. ${ }^{17}$

The deltoid detachment may affect functional recovery. ${ }^{7,8,17}$ Helical plates have been introduced to overcome this disadvantage: in this design, the plate is twisted $90^{\circ}$ to lie on the lateral aspect of the greater tuberosity and on the distal anterior side of the humeral shaft, with a parallel course along the radial nerve. ${ }^{7,16}$ In their biomechanical studies Dell'Oca ${ }^{7}$ and Krishna et al. ${ }^{16}$ showed other advantages: the gap closure at the fracture interface is better in helical plate fixation compared to straight plate fixation for all loading conditions; stress shielding is reduced due to the helical shape of the plate shifting the neutral axis into the bone. Moreover, helical plates are most useful in spiral fractures since the implant absorbs the tensile stresses caused by torsion; finally, the screws in helical plates have different directions, providing more screw-holding power than in straight plates. In 2012, Zhang et al. ${ }^{5}$ finally used a lateral distal tibial helical plate to treat proximal and middle one-third humeral fractures: according to the Constant-Murley score, $28 \%$ of patients had excellent functional outcomes, and $64 \%$ had good results.

More recently Moon and colleagues ${ }^{10}$ used pre-contoured straight humeral plates in twelve patients; they underlined the difficulty for the surgeon of proper contouring to fit the humeral shape and suggested the need of dedicated helical plates to avoid damage to the holes locking treads. At the final follow-up, patients in their study showed a Constant-Murley score of 88.6 and a shoulder abduction of $153.7^{\circ}$ on average.

Moreover, Wang et al. ${ }^{11}$ in 2018 analysed the use of $3 \mathrm{D}$ printed fracture models to fit the humeral shape accurately and their patients reached a mean Constant score of 76 at one year of follow-up.

In agreement with the aforementioned studies $^{5,10,11}$ we observed similar data in our experience: as reported in the literature, the Constant Score is influenced by the quality of reduction in the greater tuberosity, the position of the screw and plate, patient age and postoperative physiotherapy. ${ }^{5}$

In addition, satisfactory abduction and strength were evaluated in all patients at clinical follow-up: the deltoid insertion preservation may be a favorable factor that influences the patient's quality of everyday life. Generally, we think noninvasive surgery is based on gently preservation of the soft tissue rather than mini-skin incisions.

Even if we had no cases of iatrogenic radial nerve injury, it is a possible complication reported in the surgical treatment of middle third humeral fractures due to the extensive approach needed. Our suggestion is to identify and protect the nerve in those fractures with a distal diaphyseal extension; nevertheless, the helical contouring allows an anterior fixation of the plate in the distal humerus, reducing the risk of nerve damage: this feature was confirmed in 2020 by Da Silva and colleagues, ${ }^{9}$ who had no cases of iatrogenic radial nerve injury using manually-twisted plates in contrast to a $6 \%$ incidence in the straight plate group, and by the other few studies that focused on helical locking plates. ${ }^{8,10,11}$

\section{Conclusions}

The goal of surgical treatment is a stable fixation and rapid functional recovery. In our experience, humeral helical plates may be an effective surgical option for managing complex proximal third diaphyseal humeral fractures, given the deltoid muscle insertion preservation, a better plate/bone congruency and a reduced risk of radial nerve palsy, while maintaining similar healing rates and functional outcomes. The authors acknowledge the limited number of cases in this series and the findings need to be validated with a higher number of cases by different surgeons.

\section{References}

1. Nowak LL, Dehgahn N, McKee MD. 
Plate fixation for management of humerus fractures. Injury 2018;49:S338.

2. Sarmiento A, Zagorski JB, Zych GA, et al. Functional bracing for the treatment of fractures of the humeral diaphysis. J Bone Jt Surg Am 2000;82:478-86.

3. Gaebler C, McQueen MM, CourtBrown CM. Minimally displaced proximal humeral fractures: epidemiology and outcome in 507 cases. Acta Orthop Scand 2003;74:580-5.

4. Tetsworth K, Hohmann E, Glatt V. Minimally invasive plate osteosynthesis of humeral shaft fractures: current state of the art. J Am Acad Orthop Surg 2018;26:652-61.

5. Zhang L, Chen LW, Zhang W, et al. Treatment of proximal and middle onethird humeral fractures with lateral distal tibial helical plate. Eur J Orthop Surg Traumatol 2011;22:673-9.

6. Maresca A, Pascarella R, Bettuzzi C, et al. Multifocal humeral fractures. Injury 2014;45;444-7.

7. Dell'Oca AAF. The principle of helical implants. Unusual ideas worth considering. Injury 2002;33:SA1-2.
8. Yang KH. Helical plate fixation for treatment of comminuted fractures of the proximal and middle one-third of the humerus. Injury 2005;36:75-80.

9. Da Silva T, Rummel F, Knop C, et al. Comparing iatrogenic radial nerve lesions in humeral shaft fractures treated with helical or straight PHILOS plates: a 10-year retrospective cohort study of 62 cases. Arch Orthop Trauma Surg 2020;140:1931-7.

10. Moon JG, NamKwon H, Sandeep B, et al. Minimally Invasive Plate Osteosynthesis using a helical plate for metadiaphyseal complex fractures of the proximal humerus. Orthopaedics 2014;37:237-43.

11. Wang Q, Hu J, Guan J, et al. Proximal third humeral shaft fractures fixed with long helical PHILOS plates in elderly patients: benefit of pre-contouring plates on a 3D-printed model-a retrospective study. J Orthop Surg Res 2018;13:203

12. Ruedi TP, Murphy WM. AO principles of fracture management,1. Stuttgart, New York: Thieme; 2000.

13. Benninger E, Meier C. Minimally inva- sive lateral plate placement for metadiaphyseal fractures of the humerus and its implications for the distal deltoid insertion - it is not only about the radial nerve. A cadaveric study. Injury 2017;48:615-20.

14. Robinson MC, Stirling PHC, MacDonald DJ, et al. Open reduction and long locking plate fixation of complex proximal humeral metaphyseal fractures. J Bone Joint Surg Am 2020;102:2146-56.

15. Dauwe J, Grechenig P, Unterfrauner I, et al. Axillary nerve elongation in humeral fracture plating: A cadaveric study for comparison between straight and helical Philos plates. J Orthop 2020;19:233-6.

16. Krishna KR, Sridhar I, Ghista DN. Analysis of the helical plate for bone fracture fixation. Injury 2008;39:142136.

17. Gill DRJ, Torchia ME. The spiral compression plate for proximal humeral shaft nonunion: a case report and description of a new technique. J Orthop Trauma 1999;13:141-4. 